\title{
Biocompatibility Study of Collagen I Modified PCL/Gelatin/Chitosan Composite Nanofibrous Scaffold for Fibroblast and Osteoblast Cells
}

\author{
Sneh Gautam* \\ Department of Molecular Biology and Genetic Engineering, C.B.S.H, G. B. Pant University of \\ Agriculture \& Technology, Pantnagar-263145, Uttarakhand, India \\ *Corresponding author
}

\section{A B S T R A C T}

\section{Keywords}

Composite scaffold, L929 mouse fibroblasts, Human osteoblasts, Polycaprolactone, Gelatin, Chitosan, Collagen I

\section{Article Info}

Accepted: 10 September 2020 Available Online: 10 October 2020
In present study, a previously fabricated tripolymer composite scaffold of PCL/gelatin/chitosan was modified with collagen I and the modified scaffold were studied for biocompatibility of L929 mouse fibroblasts and human osteoblast cells. Phase contrast microscopy confirmed the viability of L929 mouse fibroblasts and human osteoblasts cultured with the modified composite scaffold which indicated that scaffold did not produce any toxic effect on both cells. Giemsa staining demonstrated the fibroblastic characteristic morphology of L929 and osteoblastic characteristic morphology of human osteoblast cells. FE-SEM study illustrated the adhesion and proliferation of L929 fibroblasts and osteoblasts, and retained their characteristic morphology on the modified PCL/gelatin/chitosan composite scaffold. Therefore, these results indicated that both cells were viable, proliferating, and showing their phenotypic morphology on collagen I modified PCL/gelatin/chitosan composite nanofibrouss caffold. Hence, the collagen I modified PCL/gelatin/chitosan composite nanofibrouss caffold might be an acceptable contender for skin and bone tissue engineering applications.

\section{Introduction}

Tissue engineering is an interdisciplinary field, nowadays which is being used for the development of biological substitutes and to restore, maintain, or improve the function of diseased tissue (Ma, 2008; Langer and Vacanti, 1993; Praveen, 2006). The key aspects of tissue engineering are cells, a scaffold and growth factors which play a pivotal role in tissue regeneration and tissue healing, although all the three components are essential, but scaffold plays a critical role for tissue regeneration (Ott et al., 2008; Ikada et al., 2006; Gautam and Ambwani; 2019). The main role of the scaffold in tissue engineering is to provide a biomimetic environment to the cells so that cells can proliferate and differentiate in a specific tissue (Sharma et $a l ., 2011)$. Thus, a tissue engineering scaffold should be structurally and chemically similar to the extra-cellular matrix (ECM) of our body. The extra-cellular matrix of our body mainly made up of nanoscale collagen (protein) fibers which are embedded in the gel of polysaccharides (Aumailley and Gayraud, 
1998). Therefore, a scaffold which is made up of nanosacle fibers would show architectural similarity to the ECM of our body. Recently, electrospinning is an attractive and simple technique which is used to produce continuous nanofibers for tissue engineering application (Murugan and Ramakrishna, 2007).

The electrospinning process controls the scaffold architecture at the nano and micro scales for flexible tissue design that improves cellular response and biocompatibility because of the morphological similarities to the three-dimensional ECM protein fiber network (Zong et al., 2005). It has also been seen in previous studies that electrospun scaffold possess nanoscale topography which comprises many vital properties similar to the native ECM of our body such as high porosity and very high surface area-to-volume ratio that initiates the cell adhesion, proliferation and migration (Lim and Mao, 2009; Jun et al., 2018) . Additionally, in order to fulfill the diverse need of tissue engineering, chemical composition of scaffold is also an important aspect. To mimic the chemical composition of ECM various materials have been exploited.

In previous study, a composite scaffold of PCL/gelatin/chitosan (Gautam et al., 2014) has been fabricated on the basis of excellent properties of PCL, gelatin and chitosan. Since, PCL is a synthetic polymer (Zhang et al., 2005) which possess high mechanical properties (Chen et al., 2011) but low cell adhesive properties due to its hydrophobic nature (Kim et al., 2006; Li et al., 2006; Gautam et al., 2012). On the other hand, gelatin is a natural polymer and it is denature form of collagen protein which shows good cell adhesive properties (Ghasemi-Mobarakeh et al., 2008) but low mechanical strength (Bigi et al., 2001). Further, chitosan is a natural polysaccharide and it is deacetyl form of chitin that consists of $\mathrm{N}$-acetylglucosamine and glucosamine similar to glycosaminoglycans which is a major component of natural ECM (Chen et al., 2010; Mourya et al., 2010). Chitosan exhibits good cell adhesive and proliferative properties whereas it also shows poor mechanical stability which is almost similar to gelatin (Pok et al., 2013; Albanna et al., 2013). Thus, the combination of PCL/gelatin/chitosan (Gautam et al., 2014) to fabricate an electrospun scaffold was found remarkable that mimicked the chemical composition (protein and polysaccharide) more similar to the extracellular matrix which provoked cell proliferation properties to the scaffold, and also provided the required mechanical strength.

In this study, an attempt has been made to mimic the previously fabricated scaffold chemically more similar with native ECM of skin and bone to increase the cell affinity for skin and bone tissue engineering applications. Hence, the previously fabricated PCL/gelatin/chitosan composite scaffold was modified with collagen I. Since, collagen I is a major component of natural ECM of skin and bone which bears different cell binding sites such as Arg-Gly-Asp (RGD) and GlyPhe-Hyp-Gly-GluArg (GFOGER) (Khew et al., 2007; Gautam et al., 2013) that influences adhesion mechanism of cells to the scaffold. Further, in the present study, collagen I modified tripolymer composite scaffold of PCL/gelatin/chitosan were exploited for biocompatibility study of L929 mouse fibroblasts and human Osteoblasts cell line.

\section{Materials and Methods}

Chitosan (degree of deacetylation $\geq 75 \%$ ) powder, PCL $(\mathrm{Mw}=80,000)$, Gelatin (type B bovine skin) and collagen (type I from calf skin) were purchased from Sigma-Aldrich (St. Louis, MO). Phosphate buffer saline (PBS) and Dulbecco's modified eagle medium 
(DMEM) were also procured form SigmaAldrich (St. Louis, MO). Giemsa stain, methanol and Chloroform were obtained from Fisher Scientific, Mumbai, India. Glacial acetic acid was acquired Qualigens Fine Chemical, Mumbai, India. Fetal bovine serum (FBS) was purchased from Hyclone, U.S.A. Skin fibroblast-like cells (L929) from mouse connective tissue was received from National Centre for Cell Science (NCCS Pune, India). Human osteoblast cell line was obtained from Jaslok Hospital and Research Centre, Mumbai, India. 1-ethyl-3-(3dimethylaminopropyl) carbodiimide (EDAC) was obtained from Himedia laboratories Pvt. Limited, Mumbai, India.

Fabrication of collagen I modified PCL/gelatin/chitosan composite scaffold

First of all, PCL/gelatin/chitosan composite nanofibrous scaffold was synthesized by electrospinning method and then the scaffold was modified with $1 \mathrm{wt} \%$ collagen I solution. The electrospinning of PCL/gelatin/chitosan composite nanofibrous scaffold was done according to previously reported parameters (Gautam et al., 2014). Subsequently, the fabricated electrospun PCL/gelatin/chitosan composite nanofibrous scaffold was submerged in EDAC $(10 \mathrm{mg} / \mathrm{mL}$ in PBS) solution and put at $4^{\circ} \mathrm{C}$ for $4 \mathrm{~h}$. The treatment of EDAC was given to activate carboxylic group of gelatin present in scaffold. After EDAC treatment, scaffold was treated with $1 \mathrm{wt} \%$ collagen I concentration at $4^{\circ} \mathrm{C}$ for $24 \mathrm{~h}$ (Ma et al., 2005)

Afterward, the modified scaffold was airdried overnight and the morphology of this scaffold was investigated by FE-SEM (Quanta 200F Model, FEI, Netherland). Before imaging, the gold coating was done of the modified scaffold using a sputter coater (Biotech SC005, Switzerland).
Cell culturing of fibroblasts and Osteobalsts

L929 mouse fibroblast cells were maintained in DMEM media which was supplemented with $10 \%$ FBS, penicillin (100 units $/ \mathrm{mL}$ ) and streptomycin $(100 \mu \mathrm{g} / \mathrm{mL})$. Similarly, human osteoblasts were also cultured in DMEM supplemented with penicillin (100 units/mL) and streptomycin $(100 \mu \mathrm{g} / \mathrm{mL}), 10 \%$ FBS, $2 \mu \mathrm{L} / \mathrm{mL}$ glutamine and $12 \mu \mathrm{L} / \mathrm{mL}$ insulin. Confluent mouse fibroblasts and human osteoblasts were detached from culture flask by trypsinization using $0.25 \%$ Trypsin-EDTA for 5 minutes and then suspended in complete growth medium to perform next experiments.

\section{Cell viability of fibroblasts and osteoblasts cultured with modified scaffold}

Cell viability of L929 mouse fibroblasts and human osteoblasts incubated with collagen I modified PCL/gelatin/chitosan composite nanofibrous scaffolds were demonstrated by phase contrast microscopy(Zeiss, Pvt. Ltd. Bangalore, India) for 1,3 and 5 days. The modified scaffolds were sterilized by UV treatment from both sides for 30 minutes each.

Before cell seeding, scaffolds were immersed in $100 \mu \mathrm{L}$ DMEM media in 96 wells plate overnight at $37^{\circ} \mathrm{C}$ to make scaffolds surface more efficient for cell attachment. After PBS washing, scaffolds were incubated with L929 mouse fibroblast cells and human osteoblast cells at a density of $5 \times 10^{3}$ cells/well (volume $200 \mu \mathrm{L}$ ) and $1 \times 10^{3}$ cells/well (volume $200 \mu \mathrm{L}$ ) respectively for 1,3 and 5days in $\mathrm{CO}_{2}$ incubator (BINDER, Germany). During cell culture, the media was changed every $24 \mathrm{~h}$. After incubation period, cell-scaffolds constructs were observed under the phase contrast microscopy. The experiment was performed in triplicates. 
Morphological characteristics of fibroblasts and osteoblastscultured with modified scaffold

Histological staining (Giemsa staining) was performed to investigate the morphological integrity of L929 mouse fibroblasts and human osteoblasts cultured with collagen I modified $\mathrm{PCL} /$ gelatin/chitosan composite scaffold for 1, 3 and 5 days. Scaffolds were placed in 48 well culture plate and incubated with $5 \times 10^{3}$ cells/well fibroblasts and $1 \times 10^{3}$ cells/well osteoblasts at $37^{\circ} \mathrm{C}$ in $\mathrm{CO}_{2}$ incubator. After incubation period, media was discarded and cell-scaffold construct in culture plate were fixed with $50 \%$ methanol for $1 \mathrm{~h}$ at room temperature. Consequently, giemsa stain was added into the wells of plate for $5 \mathrm{~min}$. Afterwards, cell-scaffold construct was washed with distilled water and the stained cells with scaffold were examined under phase contrast microscope.

\section{Adhesion of fibroblasts and osteoblasts cells on modified composite scaffold}

Adhesion of L929 mouse fibroblasts and osteoblasts on collagen I modified $\mathrm{PCL} /$ gelatin/chitosan composite scaffolds were demonstrated by FE-SEM. Scaffolds were cultured with $100 \mu \mathrm{L}$ DMEM media overnight in 96 well culture plate in $\mathrm{CO}_{2}$ incubator at $37^{\circ} \mathrm{C}$. After PBS washing, scaffolds were cultured with L929 fibroblasts and human osteoblasts at a density of $5 \times 10^{3}$ cells/well and $1 \times 10^{3}$ cells/well respectively for 1,3 and 5 days. After incubation period, scaffold were rinsed with PBS three times and fixed with $2.5 \%$ glutaraldehyde at $4^{\circ} \mathrm{C}$ for $6 \mathrm{~h}$.

Consequently, scaffolds were dehydrated with gradient concentration of ethanol $(50 \%, 70 \%$, $95 \%$, and $100 \%$ ) at $4{ }^{\circ} \mathrm{C}$ for 30 minutes each for FE-SEM analysis to observe the morphology of attached cell on collagen I modified PCL/gelatin/chitosan composite nanofibrous scaffold.

\section{Results and Discussion}

\section{Morphology of collagen I modified PCL/gelatin/chitosan nanofibrous scaffold composite}

The PCL/gelatin/chitosan composite scaffold was modified by immersing in collagen I (1.0 wt \%) solution as discussed above. The fiber morphology of PCL/gelatin/chitosan and collagen I modified PCL/gelatin/ chitosan composite scaffold has been analyzed by FESEM as shown in (Fig. 1). The PCL/gelatin/chitosan composite nanofibrous scaffold showed random fiber morphology with $350 \pm 55 \mathrm{~nm}$ fiber diameter and $2.5 \pm 0.59$ $\mu \mathrm{m}$ pore size [Fig.1 (a-b)]. The collagen I modified PCL/gelatin/chitosan composite scaffold also showed random fiber morphology but fibers were found collapsed to each other [Fig.1(c-d)]. The collapse fiber morphology may be arise from the immobilization of collagen I on the surface of PCL/gelatin/chitosan scaffold. The modified composite scaffold showed fiber diameter in the range $426 \pm 114 \mathrm{~nm}$ and pore size in the range $1.0 \pm 0.36 \mu \mathrm{m}$. The modified composite scaffold showed higher fiber diameter and lower pore size as compared to the unmodified scaffold which might be due to the immobilization of collagen I on the surface of PCL/gelatin/chitosan scaffold.

Cell viability of fibroblasts and osteoblasts cultured with modified composite scaffold

Phase contrast microscopy of L929 fibroblasts and human osteoblasts cultured with modified scaffold has been shown in Fig. 2 for 1, 3 and 5 days of incubation period. It is clear from Fig. 2, L929 mouse fibroblast cells cultured with collagen I modified PCL/gelatin/chitosan composite scaffold were fully viable and 
increased in density with incubation period. This indicates that any toxic substance was not leach out from modified composite scaffold or scaffold materials did not produce any toxic effect on the cells, and the cells were observed proliferating in nature with incubation period. Similar results were found with human osteoblasts. These cells were also found to increase in density with incubation period that indicated the proliferating nature of the cells with scaffold.

Fig.1 Morphology of electrospun PCL/gelatin/chitosan and collagen I modified

PCL/gelatin/chitosan composite scaffold: (a) and (b) PCL/gelatin/chitosan composite scaffold at 5,000x and 10,000x magnification; (c) and (d) collagen I modified PCL/gelatin/chitosan composite scaffold at 5,000x and 20,000x magnification respectively
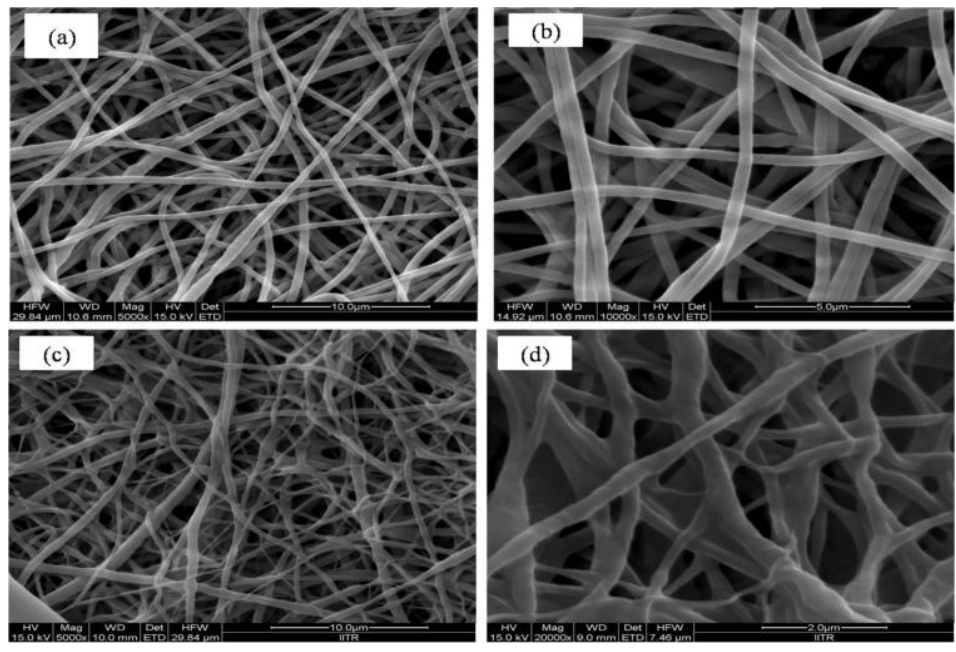

Fig.2 Phase contrast microscopy images of L929 cells and human osteoblasts incubated with collagen I modified PCL/gelatin/chitosan composite scaffold at different magnification. White arrows represent the scaffold in the wells of tissue culture plate
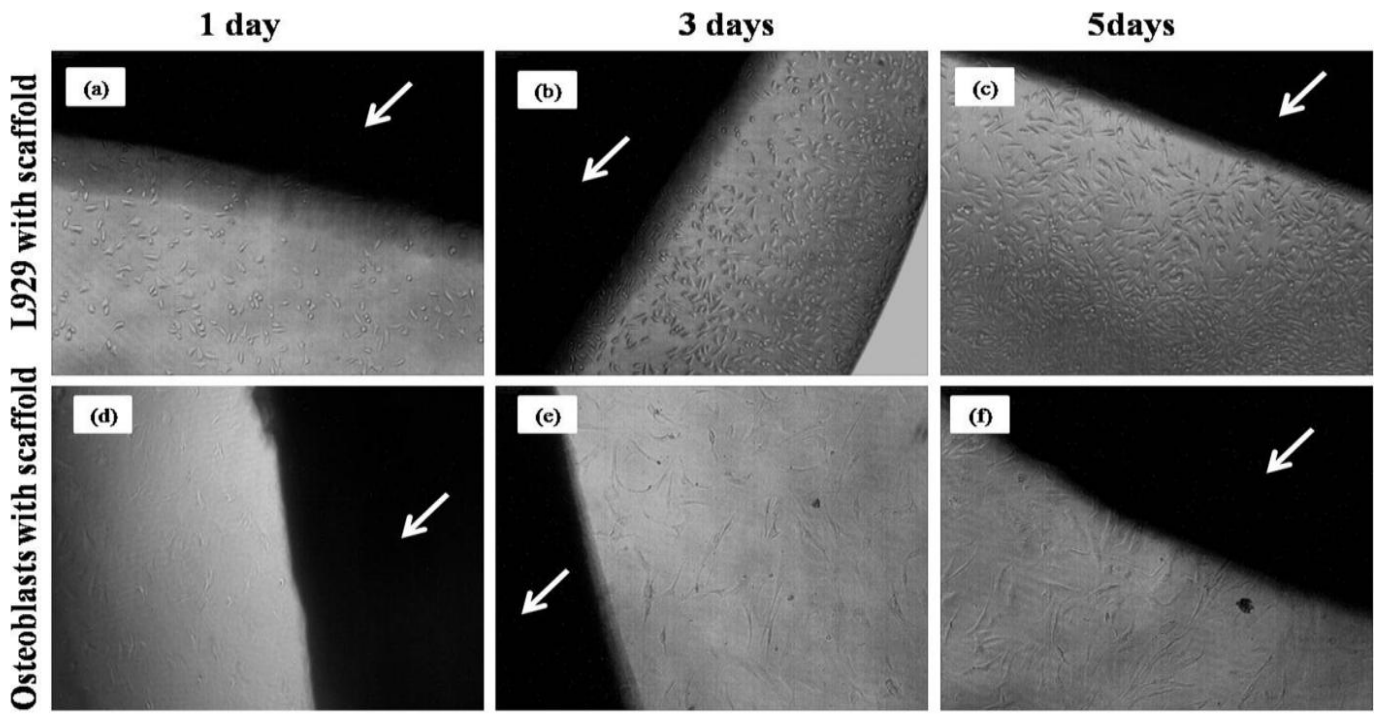
Fig.3 Morphology of osteoblast cells incubated with PCL/gelatin/collagen I composite scaffold at different magnification: arrows represent the scaffold in the wells of tissue culture plate
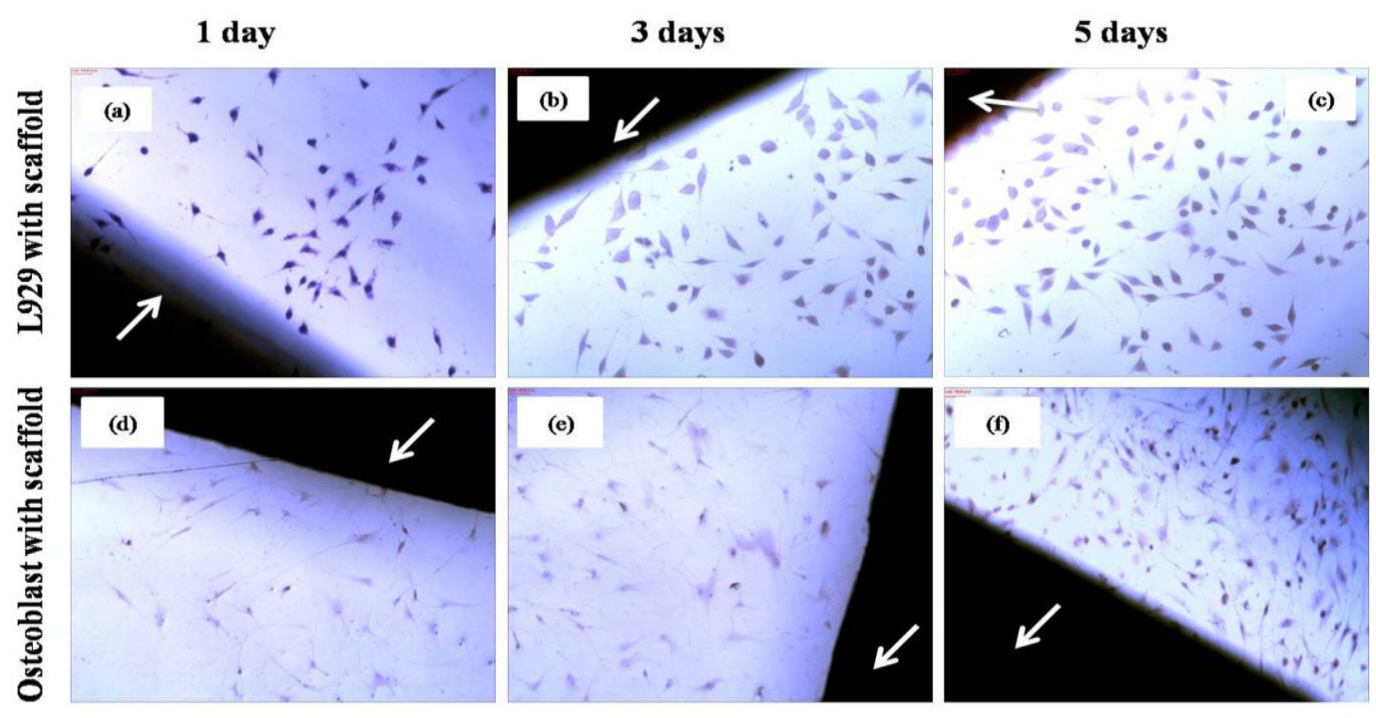

Fig.4 Adhesion of L929 and osteoblast cells on PCL/gelatin/collagen composite scaffold: (a), (b) and (c) represent L929 cell adhesion on composite scaffold after 1, 3 and 5 days respectively at 2000x magnification; (d), (e) and (f) represent L929 cell adhesion on composite scaffold after 1,

3 and 5 days respectively at 1000x magnification
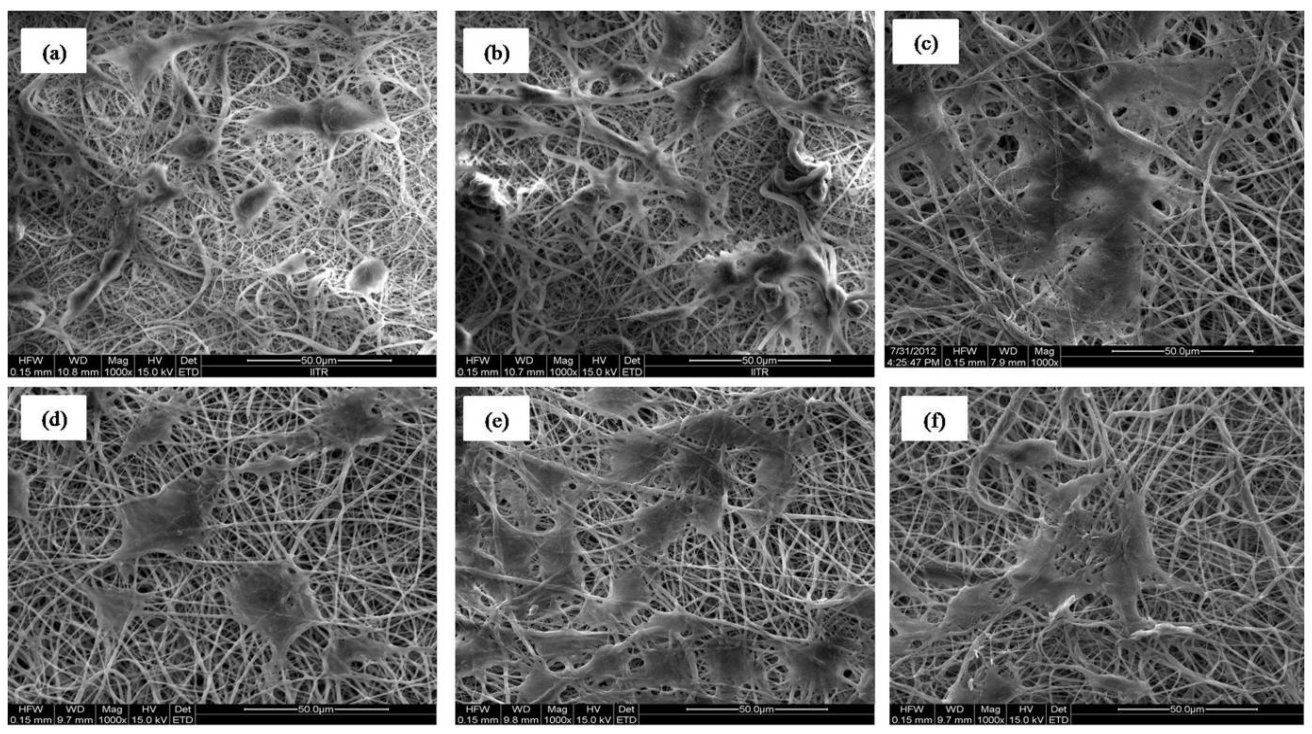

Morphology of fibroblasts and osteoblasts cultured with modified composite scaffold

Giemsa staining of L929 mouse fibroblasts and human osteoblasts was shown in Fig. 3 cultured collagen I modified with
PCL/gelatin/chitosan composite nanofibrous scaffold to study their morphology after 1, 3 and 5 days. After 1 day of cell culture, mostly L929 fibroblasts were found to alter into spindle shape morphology whereas after 3 day of incubation period, mostly cells 
converted into spindle shape morphology which is phenotypic characteristic of these cells (Fig. 3). After 5 days of cell culture also, maximum cells were observed in spindle shape morphology with increased density. This characteristic morphology of L929 fibroblasts also discussed in earlier studies (Theerakittayakorn and Bunprasert et al., 2011).Additionally, the morphology of human osteoblasts was found to alter into polygonal shape after 1 day of cell seeding. After 3 days of incubation period, mostly cells converted into polygonal morphology and after 5 days, the cells were converted prominently into polygonal morphology (Fig. 3) which is a phenotypic characteristic of these cells. Besides this, the cells were also observed in high density with incubation period. This type of morphology of osteoblast cells was also reported previously on the titanium based scaffold (Yang et al., 2002). Thus, Giemsa staining confirmed that both cells maintained their characteristic morphology in the presence of composite scaffold.

\section{Adhesion of fibroblasts and osteoblasts on modified composite scaffold}

FE-SEM images showed the adhesion of L929 mouse fibroblasts and human osteoblasts on the collagen I modified $\mathrm{PCL} /$ gelatin/chitosan composite nanofibrous scaffold (Fig. 4). After 1 day of cell culture, some L929 mouse fibroblasts were found in round morphology and few cells were observed in spindle shape. After 3 days of incubation period, they started spreading on the scaffold. Subsequently, after 5 day of cell culture, the cells were found merge with fiber morphology. Similarly, osteoblast cells were found adhere to the surface of modified composite scaffold as shown in Fig. 4. After 1 day of cell culture, the osteoblast cells started to convert into polygonal morphology over the modified scaffold. After 3 days, mostly cells converted into its characteristic polygonal morphology and polygonal shape became more prominent. After 5 days of cell culture, the osteoblast cells were found merge with fiber morphology. These results showed that the surface of scaffolds were suitable for the cells and provided a more favorable environment to the cells for adhesion and proliferation because both cells are gaining their characteristic morphology on the scaffold even after 1 day of cell culture. Thus, FE-SEM results also confirmed the L929 fibroblasts and human osteoblasts viability and proliferation, and also supported the results of Phase contrast microscopy and Giemsa staining.

In conclusions a composite nanofibrous scaffold of PCL/gelatin/chitosan was successfully modified with collagen I to enhance the cell affinity of scaffold. L929 mouse fibroblasts and human osteoblast cells were found viable when incubated with the modified composite scaffold that confirmed the biocompatibility of this scaffold for both cells. Further, mouse fibroblasts and human osteoblasts both cells maintained their phenotypic morphological characteristic throughout the culture period. FE-SEM images showed the adhesion and also the characteristic morphology of these cells on the modified composite scaffold with incubation time that confirmed the viability and proliferation of both cells on the scaffold. Thus, the biocompatibility study and FE-SEM results of modified PCL/gelatin/chitosan composite scaffold indicated that the scaffold might be a good candidate for skin and bone tissue engineering applications.

\section{Acknowledgment}

The author is grateful to IIT Roorkee for work related to the fabrication of scaffold and Jaslok Hospital and Research Centre, Mumbai for carried out biocompatibility study. 


\section{References}

Ma, P. X. 2008. Biomimetic Materials for Tissue Engineering. Advanced drug delivery reviews. 60:184-198.

Langer, R. and Vacanti, J. P. 1993. Tissue Engineering. Science.260:920-926.

Parveen, S., Krishnakumar, K. and Sahoo, S. 2006. New Era in Health Care: Tissue Engineering. 1:8-24.

Ott, H.C., Matthiesen, T.S., Goh, S.K., Black, L.D., Kren, S.M., Netoff, T.I. and Taylor, D.A. 2008. Perfusiondecellularized matrix: using nature's platform to engineer a bioartificial heart. Nature medicine. 14: 213-221.

Ikada, Y. 2006. Challenges in tissue engineering. Journal of the Royal Society Interface.3:589-601.

Sharma, C., Gautam, S., Dinda, A.K. and Mishra, N.C. 2011. Cartilage tissue engineering: current scenario and challenges. Advance Material Letter. 2:90-99.

Aumailley, M. and Gayraud, B. 1998. Structure and biological activity of the extracellular matrix. Journal of Molecular Medicine. 76:253-265.

Murugan, R. and Ramakrishna, S. 2007. Design strategies of tissue engineering scaffolds with controlled fiber orientation. Tissue Engineering.13:1845-1866.

Zong, X., Bien, H., Chung, C.Y., Yin, L., Fang, D., Hsiao, B.S., Chu, B. and Entcheva, E. 2005. Electrospun finetextured scaffolds for heart tissue constructs. Biomaterials. 26: 53305338.

Lim, S.H. and Mao, H.Q. 2009. Electrospun scaffolds for stem cell engineering. Advanced drug delivery reviews. 61:1084-1096.

Jun, I., Han, H.S., Edwards, J.R. and Jeon, H. 2018. Electrospun fibrous scaffolds for tissue engineering: Viewpoints on architecture and fabrication. International Journal of Molecular Sciences. 19:745.

Gautam, S., Chou, C. F., Dinda, A. K., Potdar, P. D. and Mishra, N. C. 2014. Fabrication and characterization of PCL/gelatin/chitosan ternary nanofibrous composite scaffold for tissue engineering applications. Journal of Material Science. 49: 1076-1089.

Zhang, Y., Ouyang, H., Chwee, T. L., Ramakrishna, S. and Huang, Z. M. 2005. Electrospinning of gelatin fibers and gelatin/PCL composite fibrous scaffolds. Journal of Biomedical Materials Research Part B: Applied Biomaterials.72: 156-165.

Honglin, C., Jin, H., Jiahui, Y., Shiyuan, L. and Ping, G. 2011. Electrospun chitosan-graft poly $(\varepsilon-$ caprolactone)/poly ( $\quad$-caprolactone) cationic nanofibrous mats as potential scaffolds for skin tissue engineering. International Journal of Biological Macromolecules. 48: 13-19.

Kim, C.H., Khil, M.S., Kim, H.Y., Lee, H.U. and Jahng, K.Y. 2006. An improved hydrophilicity via electrospinning for enhanced cell attachment and proliferation. Journal of Biomedical Materials Research Part B: Applied Biomaterials. 78: 283-290.

Li, W.J., Cooper Jr, J.A., Mauck, R.L. and Tuan, R. S. 2006. Fabrication and characterization of six electrospun poly ( $\alpha$-hydroxy ester)-based fibrous scaffolds for tissue engineering applications. Actabiomaterialia. 2: 377 385.

Gautam, S., Dinda, A. K. and Mishra, N. C. 2012.Fabrication and characterization of PCL/gelatin composite nanofibrous scaffold for tissue engineering applications by electrospinning method. Material Science and Engineering C. 33: 1228-1235. 
Ghasemi-Mobarakeh, L., Prabhakaran, M.P., Morshed, M., Nasr-Esfahani, M. H. and Ramakrishna, S. 2008. Electrospun poly ( $\varepsilon$-caprolactone)/ gelatinnanofibrous scaffolds for nerve tissue engineering. Biomaterials. 29:4532-4539.

Bigi, A., Cojazzi, G., Panzavolta, S., Rubini, K. and Roveri, N. 2001. Mechanical and thermal properties of gelatin films at different degrees of glutaraldehyde crosslinking. Biomaterials. 22: $763-$ 768.

Chen, J.S., Tu, S.L. and Tsay, R.Y. 2010. A morphological study of porous polylactide scaffolds prepared by thermally induced phase separation. Journal of the Taiwan Institute of Chemical Engineers. 41: 229-238.

Mourya, V. K., Inamdar, N. N. and Tiwari, A. 2010. Carboxymethyl chitosan and its applications. Advanced Materials Letters. 1: 11-33.

Pok, S., Myers, J.D., Madihally, S.V. and Jacot, J.G. 2013. A multilayered scaffold of a chitosan and gelatin hydrogel supported by a PCL core for cardiac tissue engineering. Actabiomaterialia. 9: 5630-5642.

Albanna, M.Z., Bou-Akl, T.H., Blowytsky, O., Walters III, H.L. and Matthew, H.W. 2013. Chitosan fibers with improved biological and mechanical properties for tissue engineering applications. Journal of the Mechanical Behavior of Biomedical Materials. 20: 217-226.
Khew, S. T. and Tong, Y. W. 2007. The specific recognition of a cell binding sequence derived from type I collagen by Hep3B and L929 cells. Biomacromolecules. 8: 3153-3161.

Ma, Z., Gao, C., Gong, Y. and Shen, J. 2005. Cartilage tissue engineering PLLA scaffold with surface immobilized collagen and basic fibroblast growth factor. Biomaterials. 26: 1253-1259.

Theerakittayakorn, K. and Bunprasert, T., 2011. Differentiation capacity of mouse L929 fibroblastic cell line compare with human dermal fibroblast. World Academy of Science, Engineering and Technology International Journal of Medical and Health Sciences. 5: 51.

Yang, Y., Tian, J., Deng, L. and Ong, J.L. 2002. Morphological behavior of osteoblast-like cells on surfacemodified titanium in vitro. Biomaterials. 23: 1383-1389.

Gautam, S., and Sonu A. 2019. Tissue Engineering: New Paradigm of Biomedicine. Biosciences Biotechnology Research Asia. 16: 521532.

Gautam, S., Chou, C.F., Dinda, A.K., Potdar, P.D. and Mishra, N.C. 2013. Surface modification of nanofibrous polycaprolactone/gelatin composite scaffold by collagen type I grafting for skin tissue engineering. Materials Science and Engineering: C. 34: 402409.

\section{How to cite this article:}

Sneh Gautam. 2020. Biocompatibility Study of Collagen I Modified PCL/Gelatin/Chitosan Composite Nanofibrous Scaffold for Fibroblast and Osteoblast Cells. Int.J.Curr.Microbiol.App.Sci. 9(10): 1017-1025. doi: https://doi.org/10.20546/ijcmas.2020.910.122 